\title{
The influence of solid-liquid interfacial energy anisotropy on equilibrium shapes, nucleation, triple lines and growth morphologies
}

\author{
M. Rappaz,* J. Friedli, A. Mariaux and M. Salgado-Ordorica \\ Laboratoire de simulation des matériaux, Ecole Polytechnique Fédérale de Lausanne, IMX-STI, Station 12, \\ CH-1015 Lausanne, Switzerland
}

Received 15 January 2010; revised 23 February 2010; accepted 24 February 2010

Available online 1 March 2010

\begin{abstract}
The anisotropy of the solid-liquid interfacial energy plays a key role during the formation of as-solidified microstructures. Using the $\xi$-vector formalism of Cahn and Hoffman, this contribution presents the effect that anisotropy has on the equilibrium shapes of crystals and on surface tension equilibrium at triple lines. Consequences for heterogeneous nucleation of anisotropic crystals and for dendritic growth morphologies are detailed with specific examples related to $\mathrm{Al}-\mathrm{Zn}$ and $\mathrm{Zn}-\mathrm{Al}$ alloys.

(c) 2010 Acta Materialia Inc. Published by Elsevier Ltd. All rights reserved.
\end{abstract}

Keywords: Solid-liquid interfacial energy anisotropy; Heterogeneous nucleation; Equilibrium shape; Dendrite morphology

\section{Introduction}

The formation of primary phase microstructures in metallic alloys at speeds of typically less than $0.01 \mathrm{~m} \mathrm{~s}^{-1}$ is primarily controlled by diffusion of solute elements in the liquid phase and capillarity effects [1]. As diffusion in the liquid phase is isotropic, the growth directions of dendrites, as well as their growth kinetics, are dictated by the anisotropy of the solid-liquid $(s-\ell)$ interfacial energy $\gamma_{s \ell}$. This anisotropy, which is typically less than a few per cent in metals, has been measured by the observation of the equilibrium shape of quenched droplets [2-4] or of the menisci at triple lines between two grains and the liquid in a thermal gradient $[5,6]$.

For simple situations such as $\left\langle\begin{array}{lll}1 & 0 & 0\end{array}\right\rangle$ dendrites in cubic metals, it is known from solvability theory and from numerical simulations based on either boundary integrals [7] or phase-field methods [8] that an increased anisotropy of the $s-\ell$ interfacial energy makes the dendrite tip sharper and grow faster. Unlike this case where the two principal radii of curvature of the dendrite tip are equal, no such fundamental theoretical basis exists for dendrites having unequal principal radii of curvature. This is typically the case for hexagonal close-packed (hcp) systems such as $\mathrm{Zn}$ or $\mathrm{Mg}$, for which dendrites grow along $\langle 1 \overline{1} 00\rangle$ and $\langle 11 \overline{2} 0\rangle$, respectively $[9,10]$. But this is also the case for the $\mathrm{Al}-\mathrm{Zn}$ system, for which recent

\footnotetext{
*Corresponding author. E-mail: michel.rappaz@epfl.ch
}

observations showed that the growth directions change with composition $[11,12]$ : at low $\mathrm{Zn}$ content, face-centered cubic (fcc) dendrites grow along the usual $\left\langle\begin{array}{lll}1 & 0 & 0\end{array}\right\rangle$ directions, but as $\mathrm{Zn}$ content is increased, there is a continuous transition toward $\left\langle\begin{array}{llll}1 & 1 & 0\end{array}\right\rangle$ dendrites. This socalled "dendrite orientation transition" (DOT) was attributed to the influence of the highly anisotropic $\mathrm{Zn}$ solute element on the low anisotropy of $\gamma_{s \ell}$ of Al.

This paper briefly summarizes the link between the anisotropy of the $s-\ell$ interfacial energy, the equilibrium shape of crystals and dendrite growth directions, in three situations involving triple lines, namely heterogeneous nucleation, dendrites growing in contact with a substrate and twinned dendrite growth.

\section{2. $\xi$-vector formalism}

Considering a cubic symmetry system, the $s-\ell$ interfacial energy $\gamma_{s \ell}(\boldsymbol{n})$, where $\boldsymbol{n}$ is the unit vector direction function of the angles $\theta$ and $\phi$, can be developed in terms of the spherical harmonics, $Y_{\ell}^{m}(\theta, \phi)$, as [13]:

$$
\begin{aligned}
\frac{\gamma_{s \ell}(\boldsymbol{n})}{\gamma_{s \ell 0}}= & 1+\eta_{4}\left[Y_{4}^{0}+\sqrt{\frac{5}{14}}\left(Y_{4}^{4}+Y_{4}^{-4}\right)\right]+\eta_{6}\left[Y_{6}^{0}-\sqrt{\frac{7}{2}}\left(Y_{6}^{4}+Y_{6}^{-4}\right)\right] \\
& +\eta_{8}\left[Y_{8}^{0}-\sqrt{\frac{14}{99}}\left(Y_{8}^{4}+Y_{8}^{-4}\right)+\sqrt{\frac{65}{198}}\left(Y_{8}^{8}+Y_{8}^{-8}\right)\right]+\cdots
\end{aligned}
$$

where $\gamma_{s \ell 0}$ is a reference value for the isotropic part and the $\eta_{i}$ s are coefficients of the development. For a 
hexagonal symmetry crystal, the anisotropy can be developed as [14]:

$\frac{\gamma_{s \ell}(\boldsymbol{n})}{\gamma_{s \ell 0}}=1+\eta_{2}^{0} Y_{2}^{0}+\eta_{4}^{0} Y_{4}^{0}+\eta_{6}^{6}\left(Y_{6}^{6}+Y_{6}^{-6}\right)$

As the $s-\ell$ interfacial energy $\gamma_{s \ell}(\boldsymbol{n})$ is known, the equilibrium shape of a crystal in contact with its liquid is given by the well-known Wulff construction [15], subsequently demonstrated by Herring [16]. Alternatively, it can be constructed using the elegant formalism of the $\xi$-vector developed by Cahn and Hoffman $[17,18]$. The $\boldsymbol{\xi}_{s \ell}$-vector is defined with respect to $\gamma_{s \ell}(\boldsymbol{n})$ as:

$\boldsymbol{\xi}_{s \ell}=\nabla\left[\gamma_{s \ell}(\boldsymbol{n}) r\right]=\gamma_{s \ell} \boldsymbol{n}+\left(\frac{\partial \gamma_{s \ell}}{\partial \theta}\right)_{\max } \tau_{\max }$

where $\tau_{\max }$ is the vector perpendicular to $\boldsymbol{n}$ along which the derivative of $\gamma_{s \ell}$ is maximum. Thus, the normal component, $\boldsymbol{\xi}_{s \ell} \cdot \boldsymbol{n}$, is equal to $\gamma_{s \ell}$, whereas the in-plane component is given by $\left(\boldsymbol{\xi}_{s \ell}-\gamma_{s \ell} \boldsymbol{n}\right)$ (see Fig. 1a). Cahn and Hoffman showed that the set of vectors $\boldsymbol{\xi}_{s \ell}(\boldsymbol{n})$ (which are not parallel to $\boldsymbol{n}$ ) defines a surface which is homothetic to the equilibrium shape crystal.

The same formalism allows the surface tension $\sigma_{s \ell}$ acting on a triple line of unit vector $\boldsymbol{l}$ (Fig. 1b) to be calculated as follows:

$\boldsymbol{\sigma}_{s \ell}=\boldsymbol{\xi}_{s \ell}(\boldsymbol{n}) \times \boldsymbol{l}$

and therefore yields the wetting conditions of an anisotropic crystal on a foreign and flat substrate. Taking the equilibrium shape given by the $\xi$-plot and considering the normal direction $\boldsymbol{n}_{s f}$ of the foreign surface, the wetting condition is obtained by cutting the $\xi$-plot with a plane of normal $\boldsymbol{n}_{s f}$ displaced by $\Delta \gamma\left(\boldsymbol{n}_{s f}\right)=\left(\gamma_{\ell f}\left(\boldsymbol{n}_{s f}\right)-\right.$ $\left.\gamma_{s f}\left(\boldsymbol{n}_{s f}\right)\right)$ from the center of the particle. If the substrate is amorphous, $\gamma_{\ell f}$ is isotropic but the anisotropy of $\gamma_{s f}$ remains due to the crystal itself. While this construction is general, regardless of the type of substrate, it is shown schematically in Figure 2 for a Zn-type crystal in a 2$\mathrm{D}$ cross-section, when the $\eta_{6}^{6}$-contribution is neglected in Eq. (2). It was further assumed that $\Delta \gamma$ is isotropic, i.e. the wetting condition for any arbitrary orientation $\boldsymbol{n}_{s f}$ of the crystal on the surface is given by cutting the $\xi$-plot (in this case a lentil-type) by the tangent plane to a sphere of radius $\Delta \gamma$. Such a construction is shown for two angles $\alpha$ between the $c$-axis of the crystal and $\boldsymbol{n}_{s f}$. As can be seen, the wetting angle along the triple line

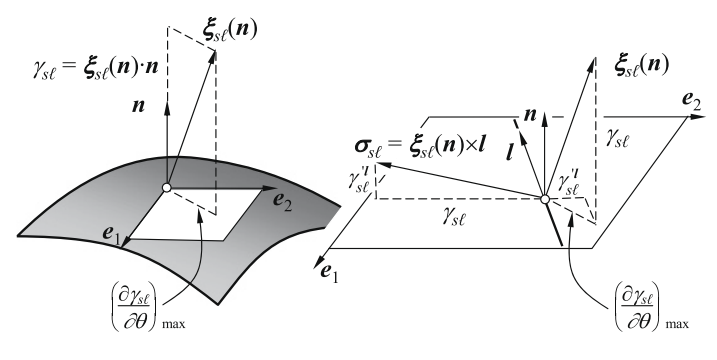

(a)

(b)

Figure 1. (a) The $\xi$-vector formalism used to calculate the equilibrium shape of a crystal. Note that the unit direction $\boldsymbol{n}$ is not normal to the surface of the $\gamma$-plot (grey area). (b) Force acting on a triple line. (After Cahn and Hoffman $[17,18]$.) (point in 2-D) is no longer constant for an arbitrary orientation of the crystal on the foreign substrate and this has several implications on both heterogeneous nucleation and growth.

\section{Heterogeneous nucleation and growth of zinc grains}

While Cahn and Hoffman have treated the case of wetting of anisotropic crystals on a flat substrate $[17,18]$, the consequences it can have on heterogeneous nucleation have not been much addressed [19]. As the equilibrium shape and the undercooling are fixed, the formation of a critical nucleus on a heterogeneous flat surface is a function of the orientation $\boldsymbol{n}_{s f}$ of the substrate with respect to the crystal only. As can be seen in Figure 2, under good wetting conditions, i.e. $\Delta \gamma>0$, the volume of the crystal is minimal for $\alpha=0$. This is directly reflected in the associated nucleation energy barrier $\Delta G_{\mathrm{c}}^{\text {het }}(\Delta T, \alpha)$, normalized by the homogeneous nucleation barrier $\Delta G_{\mathrm{c}}^{\mathrm{hom}}(\Delta T)$, as shown in Figure 3 for $\mathrm{Zn}$ [14]. For good wetting conditions, this ratio increases fairly sharply with $\alpha$, thus indicating that it is more advantageous energetically to nucleate the crystal in the basal configuration, i.e. with $\boldsymbol{c}$ parallel to $\boldsymbol{n}_{s f}$.

For $\alpha \neq 0$, the wetting angle along the solid-liquidsubstrate triple line is not constant (see Fig. 2). This also has implications on the growth of such a solid. Figure 4 shows $\mathrm{Zn}-11.3$ at.\% Al dendrites calculated with the phase-field method for three wetting conditions on the upper surface of the domain [14]. The anisotropy of $\gamma_{s \ell}$ was set with the following parameters: $\eta_{2}^{0}=-1.026$, $\eta_{4}^{0}=0.195$ and $\eta_{6}^{6}=-0.04$. The $c$-axis is pointing toward the viewer and $\boldsymbol{n}_{s f}$ is vertical, while the angle between $\boldsymbol{n}_{s f}$ and $[1 \overline{1} 00]$ is $30^{\circ}$. For the case of a neutral wetting condition (i.e. $\Delta \gamma=\left(\gamma_{\ell f}-\gamma_{s f}\right)=0$; Fig. 4a), $\langle 1 \overline{1} 00\rangle$ dendrites grow in the volume of the domain, as expected for $\mathrm{Zn}$. Note that the dendrite arm growing toward the bottom surface is rapidly slowed down as the solute layer interacts with this boundary. The growth of dendrite arms along the $c$-axis, i.e. perpendicular to the page, is almost nil. Changing the wetting condition to $\Delta \gamma=0.25 \gamma_{s \ell 0}$ (Fig. 4b) induces the growth of a new branch, in this case [2 $\overline{1} \overline{1} 0]$, along the upper boundary. This can be understood by the fact that the wetting angle at the triple line is reduced, thus allowing the solute to be rejected more easily along this direction. For $\Delta \gamma=0.5 \gamma_{s \ell 0}$, the solid wets the upper boundary even

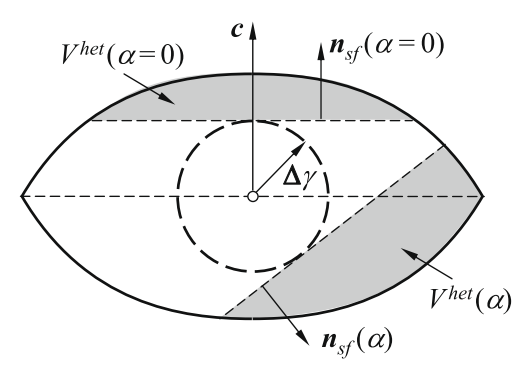

Figure 2. Effective volume of an anisotropic crystal forming on an isotropic and flat heterogeneous substrate surface at a fixed undercooling $\Delta T$ for two orientations $\alpha$ of the crystal on the substrate. (After Mariaux [14].) 


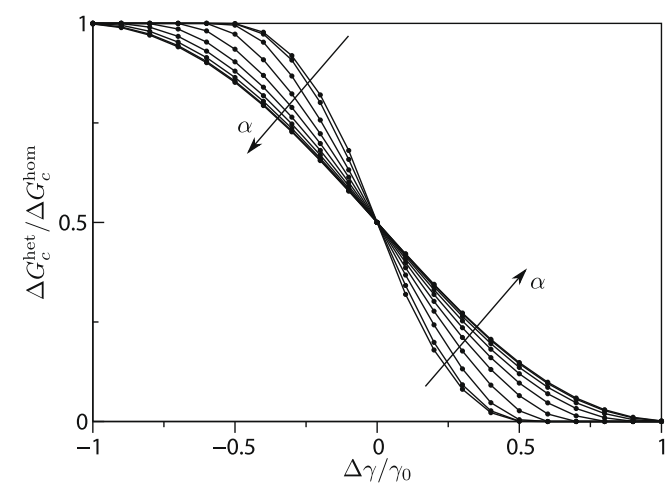

Figure 3. Nucleation Gibbs free energy barrier of a hexagonal crystal forming on an isotropic and flat substrate surface as a function of $\Delta \gamma /$ $\gamma_{s \ell 0}=\left(\gamma_{\ell f}-\gamma_{s f}\right) / \gamma_{s \ell 0}$ for various orientations of the nucleus measured by the angle $\alpha$ between the $c$-axis and the normal to the substrate surface. (From Mariaux [14].)

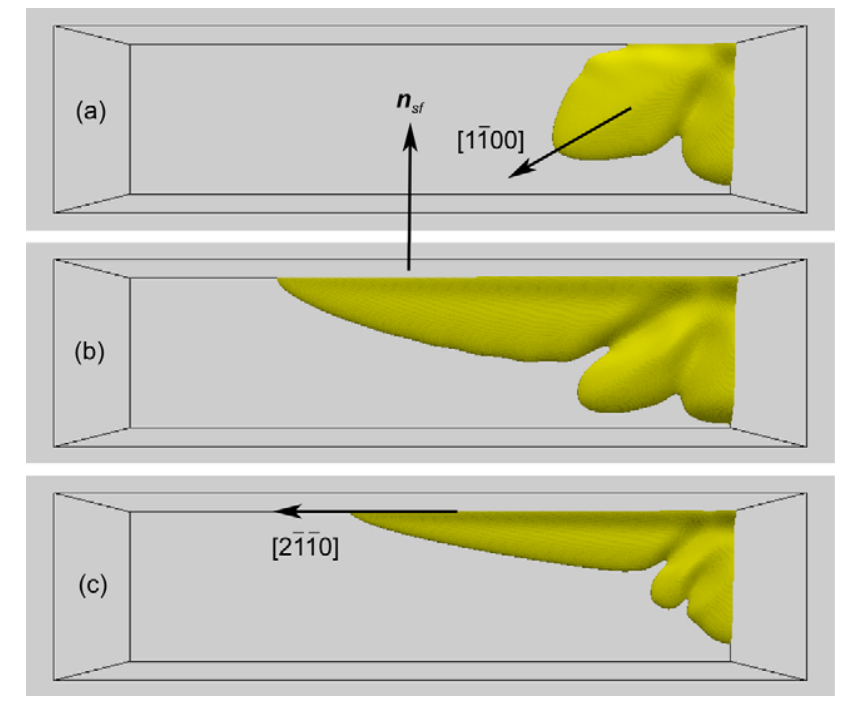

Figure 4. Phase-field computations of dendritic $\mathrm{Zn}-11.3$ at. $\% \mathrm{Al}$ growth morphologies in a confined domain with various boundary conditions on the upper surface. The $c$-axis is perpendicular to $\boldsymbol{n}_{s f}$ and pointing toward the viewer, while the $\left[\begin{array}{lll}1 & 1 & 0\end{array}\right]$ direction makes an angle of $30^{\circ}$ with the upper surface. The undercooling is $2 \mathrm{~K}$ and the wetting conditions are: (a) neutral boundary, $\Delta \gamma / \gamma_{s \ell 0}=0, t=0.18 \mathrm{~s}$; (b) $\Delta \gamma /$ $\gamma_{s \ell 0}=0.25, t=0.3 \mathrm{~s}$; (c) $\Delta \gamma / \gamma_{s \ell 0}=0.5, t=0.13 \mathrm{~s}$. (From Mariaux [14].)

better, thus producing a very edgy dendrite tip which can reject solute even more easily. For a fixed undercooling, this increases the growth speed compared to case (b) (note the time difference for Fig. $4 b$ and c). This clearly shows that the growth conditions at the triple line between solid, liquid and upper boundary are directly influenced by the wetting angle, i.e. by the interfacial energies, since this modifies the ability with which the solid can reject solute elements in the bulk.

Heterogeneous nucleation and growth of anisotropic $\mathrm{Zn}$ is responsible for some of the textures seen in hot dip galvanized coatings on steel sheets [14,20,21]. In this process, a clean pre-heated steel sheet is immersed in a $\mathrm{Zn}-0.2$ wt.\% Al bath. At the exit of the bath, air knives are adjusted to give coatings $10-20 \mu \mathrm{m}$ thick on both sides of the sheet, which then solidify in a cooling tower. Electron back-scattered diffraction (EBSD) measure- ments of such coatings typically give reconstructed maps as shown in Figure 5a. The color index measures the angle $\alpha$ between the $c$-axis and the normal $\boldsymbol{n}_{s f}$ to the sheet: as can be seen, there is a very large proportion of basal grains (blue and green grains) - in fact much larger than that expected for a random distribution of the $c$-axis. This is evidenced in the statistics of the angle $\alpha$ shown in Figure 5b, in which two distributions of nuclei orientations (random and basal) have been set so as to match best the experiments $[14,20,21]$. Not only is the proportion of basal grains larger in such coatings, but their surface fraction (i.e. texture) is further increased by the faster growth kinetics of $\langle 1 \overline{1} 00\rangle$ dendrite arms when these directions are parallel to the coating surface $[14,20]$.

\section{Dendrite morphologies}

The equilibrium shape crystals, as well as the $\gamma$-plots, reflect only weakly the anisotropy of $\gamma_{s \ell}$. Nevertheless, the mean curvature of such shapes (or of the $\xi$-plots) strongly amplifies this anisotropy since it involves second derivatives of $\gamma_{s \ell}$ [16]. As dendrites arise from a destabilization of the equilibrium shape, they should originate from the most highly curved parts and grow along those directions. The $\mathrm{Al}-\mathrm{Zn}$ alloy system is interesting since the anisotropy of $\gamma_{s \ell}$ of $\mathrm{Al}$ is weak, typically $\eta_{4} \cong 0.01$ [4], while that of $\mathrm{Zn}$ is high (see above) [2,3]. Furthermore, the high-temperature solubility limit of $\mathrm{Zn}$ in the fcc structure is high (up to 67 at. $\%)$.

It has been shown that, below about $20 \mathrm{wt} . \% \mathrm{Zn}$, fcc $\mathrm{Al}$ dendrites grow along $\left\langle\begin{array}{lll}1 & 0 & 0\end{array}\right\rangle$ directions, as do almost all cubic metals. The microstructure of quenched Al$10 \mathrm{wt} . \% \mathrm{Zn}$ dendrites grown in a Bridgman furnace and then observed in X-ray tomography is shown in Figure 6a. From the reconstructed 3-D microstructure, three sections were made on the computer, one perpendicular to the thermal gradient, and two parallel to ( $\left.\begin{array}{lll}1 & 0 & 0\end{array}\right)$ and $\left(\begin{array}{lll}0 & 1 & 0\end{array}\right)$ planes. As can be seen, the dendrite trunks and arms have indeed grown along $\left\langle\begin{array}{llll}1 & 0 & 0\end{array}\right\rangle$ directions. The $\xi$-plot or equilibrium shape corresponding to such a situation is shown in Figure 6c (the parameters are listed in the caption) $[22,23]$. Under the same solidification conditions, an Al-90 wt.\% $\mathrm{Zn}$ alloy shows different growth directions: the two longitudinal planes are now $(1 \overline{1} 1)$ and $(11 \overline{1})$ planes, making an angle of $109.5^{\circ}$ and having a common [ $\left[\begin{array}{lll}0 & 1 & 1\end{array}\right]$ direction nearly aligned with the thermal gradient. This corresponds to the direction of the dendrite trunk, while side arms also grow at $60^{\circ}$ from the trunk in each plane along $\left\langle\begin{array}{lll}1 & 1 & 0\end{array}\right\rangle$ directions. Figure $6 \mathrm{~d}$ shows the corresponding equilibrium shape. This confirms earlier measurements of Gonzales and Rappaz [11] which showed that $\left\langle\begin{array}{lll}1 & 1 & 0\end{array}\right\rangle$ dendrites grew in $\mathrm{Al}-\mathrm{Zn}$ for compositions higher than 60 wt. $\%$. Between 20 and 60 wt. $\%$, a gradual switch of the dendrite growth direction, from $\left\langle\begin{array}{llll}1 & 0 & 0\end{array}\right\rangle$ to $\left\langle\begin{array}{llll}1 & 1 & 0\end{array}\right\rangle$, is observed. This is attributed to a dependence, $\eta_{4}\left(C_{0}\right)$ and $\eta_{6}\left(C_{0}\right)$, of the weakly anisotropic $\gamma_{s \ell}$ of the fcc phase on the composition $C_{0}$ of the highly anisotropic $\mathrm{Zn}$ solute element. At intermediate compositions, interesting seaweed structures having an $\langle h k 0\rangle$ texture have been 
(a)

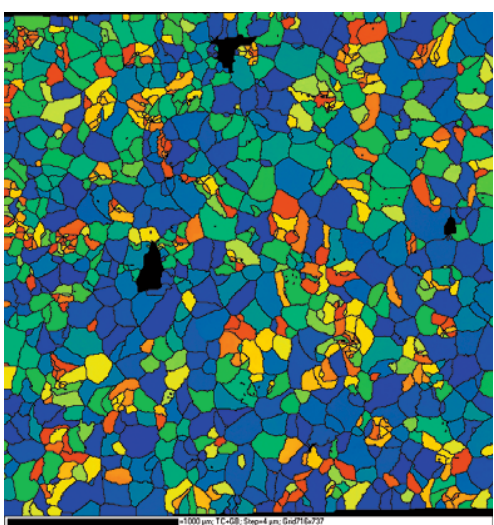

(b)

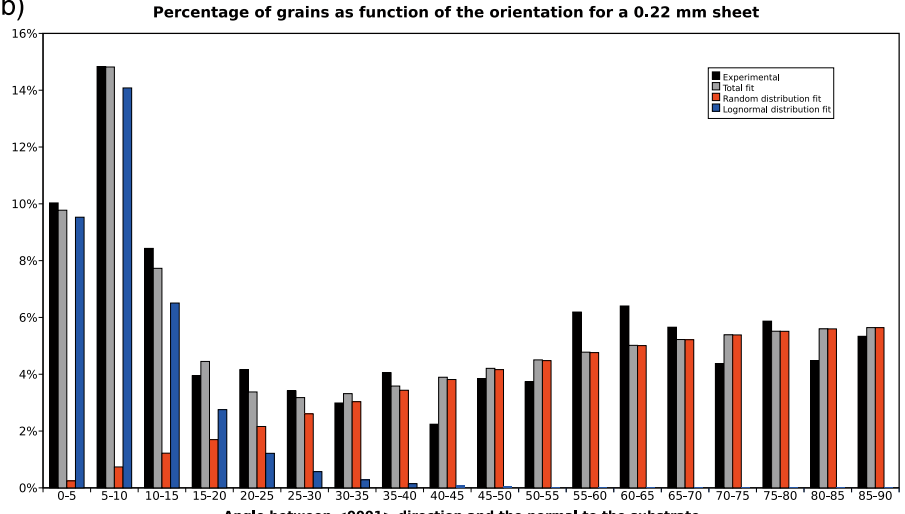

Figure 5. Preferred basal orientation of Zn crystals forming in hot-dipped galvanized coatings on a steel sheet: (a) reconstructed EBSD map of the Zn grains with a color scale from blue to red measuring the angle $\alpha$ between the $c$-axis and the normal to the coating surface (the size of the square domain is $3 \mathrm{~mm}$ ); (b) orientation distribution of the $c$-axis measured (in black), and fitted with two distributions describing the proportion of basal grains (in blue) and random grains (in red). (For interpretation of the references to color in this figure legend, the reader is referred to the web version of this article.) (From Mariaux [14].)

(a)

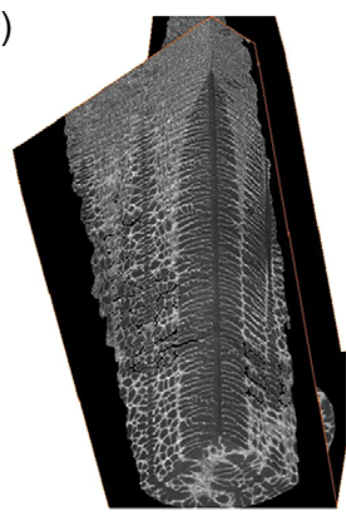

(c)

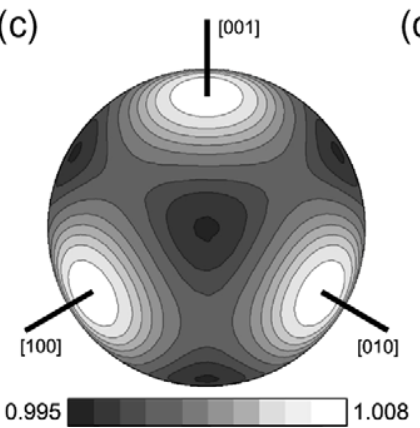

(b)

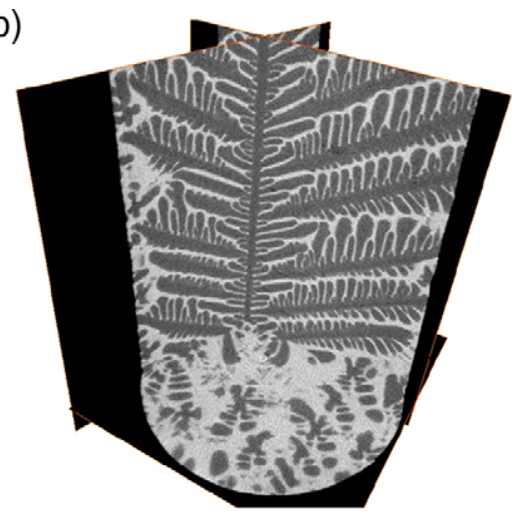

(d)

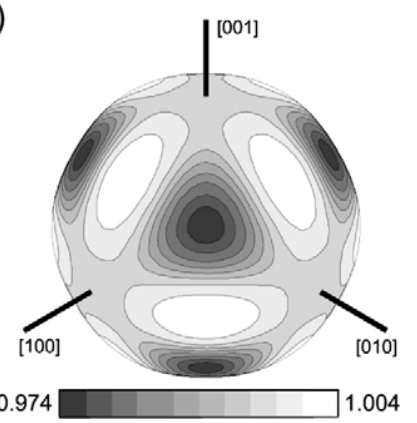

Figure 6. Transverse and longitudinal sections of a 3-D X-ray tomography reconstruction of quenched Al-Zn dendrites solidified in a Bridgman furnace at $4 \mathrm{~mm} \mathrm{~min}^{-1}$, showing the growth of (a) $\langle 100\rangle$ dendrites in Al-10 wt.\% $\mathrm{Zn}$ and (b) $\left\langle\begin{array}{llll}1 & 1 & 0\end{array}\right\rangle$ dendrites in Al-90 wt.\% Zn, respectively. The thermal gradient direction is parallel to the longitudinal sections. (c and d) The corresponding $\xi$-plots (or equilibrium shapes), calculated by Mathematica ${ }^{\circledR}[22]$ for a cubic symmetry crystal with two sets of parameters: (c) $\eta_{4}=0.0095$ and $\eta_{6}=\eta_{8}=0$, giving maximum mean curvature along $\left\langle\begin{array}{lll}0 & 0\end{array}\right\rangle$ directions; (d) $\eta_{4}=0.0147, \eta_{6}=-0.0101$ and $\eta_{8}=0$ giving maximum mean curvature along $\langle 110\rangle$ directions. The grey scale indicates the value of the $\xi$-module. (From Friedli et al. [23].)

observed [11] and are being further examined by X-ray tomography.

Under high thermal gradients and in the presence of melt convection, $\mathrm{Al}-\mathrm{Zn}$ alloys, as many other $\mathrm{Al}$ alloys, can exhibit twinned dendrites. Such microstructures, first observed more than 60 years ago [24], have a $\left[\begin{array}{lll}0 & 1 & 1\end{array}\right]$ dendrite trunk, split in its center by a $\left(\begin{array}{lll}1 & 1 & \overline{1}\end{array}\right)$ twin plane. While many conjectures remain concerning the formation of these structures (e.g. the role of convection on the nucleation of stacking faults, the change in growth direction, etc.), one issue is linked to the dendrite tip shape and to its growth advantage over regular dendrites. Some authors suggested a grooved tip [25], others an edgy tip if one takes into account the anisotropy of 


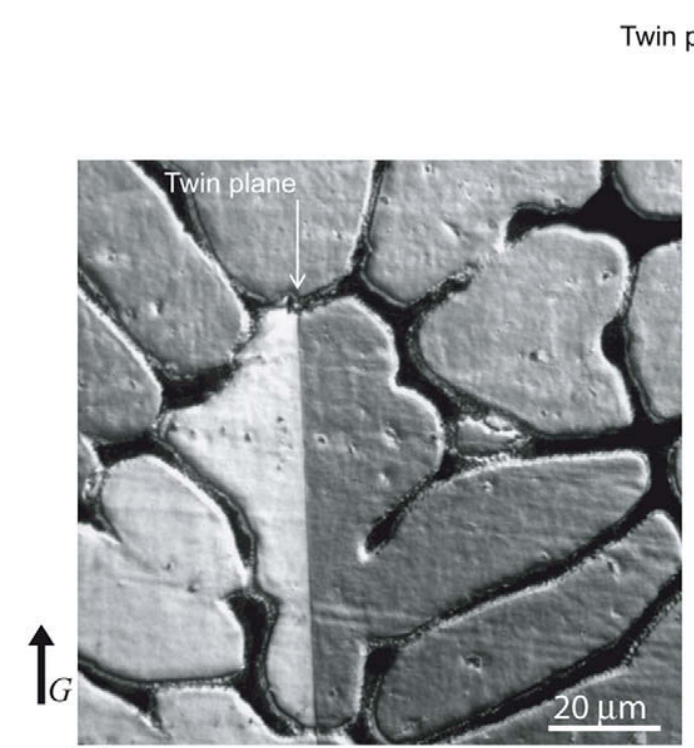

(a)

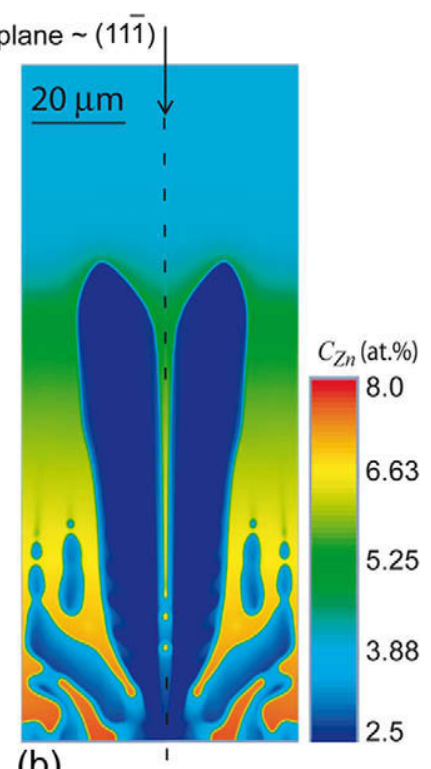

(b)

Figure 7. (a) Longitudinal section of a quenched $\left\langle\begin{array}{lll}1 & 1 & 0\end{array}\right\rangle$ twinned dendrite in an Al-20 wt.\% $\mathrm{Zn}$ alloy and (b) phase-field calculation of such a morphology with an imposed dihedral angle at the triple line between the twinned solid, untwinned solid, and liquid in an Al-5 wt.\% $\mathrm{Zn}$ alloy. (From Salgado-Ordorica [29].)

the interfacial energy [26], while more recently a doublon-tip morphology was suggested [27-29]. In this last case, the small groove necessary to satisfy the YoungLaplace equation at the triple line between the twinned solid, untwinned solid, and liquid regions is supposed to induce solute pile-up during growth and the formation of a deep and narrow liquid channel at the center of the dendrite trunk.

Figure $7 \mathrm{a}$ shows the tip of a quenched twinned dendrite in $\mathrm{Al}-20 \mathrm{wt} . \% \mathrm{Zn}$, with the twinned and untwinned parts of the dendrite appearing with different grey levels. The grooved shape of this dendrite tip definitely rules out the assumption of an edgy tip [26] and is more compatible with the weak anisotropy of $\gamma_{s \ell}$ of Al alloys. However, this experiment cannot differentiate the two other conjectures, since a thin liquid channel would definitely close during the quench. Two facts nonetheless support the doublon conjecture: (i) energy-dispersive $\mathrm{X}$-ray composition measurements near the twin plane indicate a fairly large and positive microsegregation [27,29]; (ii) 3-D phase-field simulations show that the grooved tip quickly degenerates into a doublon when appropriate boundary conditions are set at the triple line. Figure $7 \mathrm{~b}$ shows a composition map in a longitudinal section for a $\left\langle\begin{array}{llll}1 & 1 & 0\end{array}\right\rangle$ twinned dendrite of an $\mathrm{Al}-$ 5 wt. $\% \mathrm{Zn}$ alloy. In fact, only half of the dendrite was calculated with the Young-Laplace condition at the triple line being imposed as a boundary condition, and the other half was symmetrically duplicated. The figure clearly shows the formation of a $\left\langle\begin{array}{llll}1 & 1 & 0\end{array}\right\rangle$ doublon, with a solute enrichment of the liquid contained in the narrow channel until coalescence of the twinned and untwinned parts of the dendrite occurs (the twin boundary is still attractive, since $\gamma_{\text {twin }}<2 \gamma_{s \ell}$ [30]). This again shows that the condition along the triple line between twinned solid, untwinned solid, and liquid has a direct influence on the growth morphology since it affects the way the (split) dendrite tip can reject and diffuse solute.

\section{Conclusion}

The anisotropy of the solid-liquid interfacial energy plays a key role in the formation of a primary phase in various situations involving triple lines during the solidification of metallic alloys. It dictates the shape of the critical nucleus and therefore can induce a preferential orientation nucleation on a heterogeneous substrate when the anisotropy is high, e.g. for metals such as $\mathrm{Zn}$. Since dendrites emerge from the most highly curved parts of an equilibrium shape, it also influences directly their growth directions (and growth kinetics). The wetting condition at a triple line between solid, liquid and foreign substrate directly influences the dendrite growth morphology, direction and kinetics, since it affects the solute profile around it. As wetting of the solid on the foreign substrate is improved, dendrites grow with a sharper tip and thus reject solute more easily. For very good wetting, this can even change the dendrite growth direction. Similarly, twinned dendrites in Al alloys also exhibit a triple line between twinned solid, untwinned solid, and liquid. Phase-field computations show that the Young-Laplace condition at the tip induces a groove which quickly degenerates during solidification into a doublon structure due to solute pile-up in this region.

\section{Acknowledgements}

The authors would like to acknowledge the financial support of the Swiss National Fund for Scientific Research (Grant No. 200020-113260) and of the company Arcelor-Mittal. They would like to thank also Jean-Luc Desbiolles for his invaluable help in building the massively parallel version of the phase-field code. All electron microscopy measurements were obtained at the Centre Interdisciplinaire de Microscopie Electronique 
(CIME) of the Ecole Polytechnique Fédérale de Lausanne (EPFL), while X-ray tomography experiments were performed at the Swiss Light Source (SLS) of the Paul-Scherrer Institute (PSI), Villigen.

\section{References}

[1] J.A. Dantzig, M. Rappaz, Solidification, EPFL Press and CRC Press, Lausanne, 2009.

[2] W.A. Miller, G.A. Chadwick, Proc. R. Soc. A312 (1969) 257.

[3] A. Passerone, N. Eustathopoulos, Acta Metal. 30 (1982) 1349.

[4] R.E. Napolitano, S. Liu, Phys. Rev. B70 (2004) 214103.1.

[5] D.R.H. Jones, G.A. Chadwick, Philos. Mag. 22 (1970) 291.

[6] M. Gündüz, J.D. Hunt, Acta Metal. 33 (1985) 1651.

[7] Y. Saito, G. Goldbeck-Wood, H. Müller-Krumbhaar, Phys. Rev. A36 (1988) 2148.

[8] A. Karma, W.-J. Rappel, J. Cryst. Growth 174 (1997) 54.

[9] F. Weinberg, B. Chalmers, Can. J. Phys. 30 (1952) 488.

[10] K. Pettersen, N. Ryum, Metall. Trans. A20 (1989) 847.

[11] F. Gonzales, M. Rappaz, Metall. Mater. Trans. A37 (2006) 2797.

[12] T. Haxhimali, A. Karma, F. Gonzales, M. Rappaz, Nat. Mater. 4 (2006) 660.

[13] S.-K. Chan, H.-H. Reimer, M. Kahlweit, J. Cryst. Growth 32 (1976) 303.

[14] A. Mariaux, Ph.D. Thesis, Ecole Polytechnique Fédérale de Lausanne, 2010.
[15] G. Wulff, Z. Krystallogr. 34 (1901) 449.

[16] C. Herring, in: W.E. Kingston (Ed.), The Physics of Powder Metallurgy, McGraw-Hill, New York, 1951, pp. 143-179 (Chapter 8).

[17] D.W. Hoffman, J.W. Cahn, Surf. Sci. 31 (1972) 368.

[18] J.W. Cahn, D.W. Hoffman, Acta Metal. 22 (1974) 1205.

[19] J.E. Taylor, J.W. Cahn, J. Electron. Mater. 17 (1988) 443.

[20] A. Sémoroz, J. Strezov, M. Rappaz, Metall. Mater. Trans. A33 (2002) 2685.

[21] A. Sémoroz, Ph.D. Thesis \#2427, Ecole Polytechnique Fédérale de Lausanne, 2001.

[22] J. Friedli, in: Wolfram Library Archive, Representation of Equilibrium Shapes, $\gamma$-Plots and Stiffness, 2007. Available from: $<$ http://library.wolfram.com/infocenter/MathSource/7036/>

[23] J. Friedli, A. Mariaux, F. Gonzales, M. Rappaz, in: S.L. Cockcroft, D.M. Maijer (Eds.), 12th Conference on Modeling of Casting, Welding and Advanced Solidification Processes, TMS, Warrendale, PA, 2009, pp. 627-634.

[24] J. Herenguel, Rev. Metall. 45 (1948) 139.

[25] J.A. Eady, L.M. Hogan, J. Cryst. Growth 23 (1974) 129.

[26] H.J. Wood, J.D. Hunt, P.V. Evans, Acta Mater. 45 (1997) 569.

[27] S. Henry, Ph.D. Thesis \#1943, Ecole Polytechnique Fédérale de Lausanne, 1999.

[28] M. Rappaz, A. Sémoroz, S. Henry, in: International Conference on Solidification Science and Processing, Science Publishing, Inc., Enfield, NH, 2001, pp. 3-12.

[29] M. Salgado-Ordorica, Ph.D. Thesis \#4568, Ecole Polytechnique Fédérale de Lausanne, 2010.

[30] M. Rappaz, A. Jacot, W.J. Boettinger, Metall. Mater. Trans. A34 (2003) 467. 